\title{
The ALHAMBRA survey: tight dependence of the optical mass-to-light ratio on galaxy colour up to $z=1.5^{\star}$
}

\author{
C. López-Sanjuan ${ }^{1}$, L. A. Díaz-García ${ }^{1}$, A. J. Cenarro ${ }^{1}$, A. Fernández-Soto ${ }^{2,3}$, K. Viironen ${ }^{1}$, A. Molino ${ }^{4,5}$, \\ N. Benítez ${ }^{5}$, D. Cristóbal-Hornillos ${ }^{1}$, M. Moles ${ }^{1}$, J. Varela ${ }^{1}$, P. Arnalte-Mur ${ }^{6,7}$, B. Ascaso ${ }^{8}$, F. J. Castander ${ }^{9,10}$, \\ M. Cerviño ${ }^{11,5,12}$, R. M. González Delgado ${ }^{5}$, C. Husillos ${ }^{5}$, I. Márquez ${ }^{5}$, J. Masegosa ${ }^{5}$, A. Del Olmo ${ }^{5}$, \\ M. Pović ${ }^{13,5}$, and J. Perea ${ }^{5}$ \\ ${ }^{1}$ Centro de Estudios de Física del Cosmos de Aragón, Unidad Asociada al IAA-CSIC, Plaza San Juan 1, 44001 Teruel, Spain \\ e-mail: clsj@cefca.es \\ 2 Instituto de Física de Cantabria (CSIC-UC), 39005 Santander, Spain \\ 3 Unidad Asociada Observatorio Astronómico (IFCA-UV), 46980 Paterna, Spain \\ ${ }^{4}$ Universidade de São Paulo, Instituto de Astronomia, Geofísica e Ciências Atmosféricas, Rua do Matão 1226, 05508-090 São \\ Paulo, Brazil \\ 5 Instituto de Astrofísica de Andalucía (IAA-CSIC), Glorieta de la astronomía s/n, 18008 Granada, Spain \\ ${ }^{6}$ Observatori Astronòmic, Universitat de València, C/Catedrático José Beltrán 2, 46980 Paterna, Spain \\ 7 Departament d'Astronomia i Astrofísica, Universitat de València, 46100 Burjassot, Spain \\ 8 APC, AstroParticule et Cosmologie, Université Paris Diderot, CNRS/IN2P3, CEA/lrfu, Observatoire de Paris, Sorbonne Paris \\ Cité, 10 rue Alice Domon et Léonie Duquet, 75205 Paris Cedex 13, France \\ 9 Institut of Space Sciences (ICE, CSIC), Campus UAB, Carrer Can Magrans, s/n, 08193 Barcelona, Spain \\ ${ }^{10}$ Institut d'Estudis Espacials de Catalunya (IEEC), 08193 Barcelona, Spain \\ 11 Centro de Astrobiología (CSIC/INTA), 28850 Torrejón de Ardoz, Madrid, Spain \\ 12 Instituto de Astrofísica de Canarias, Vía Láctea s/n, La Laguna, 38200 Tenerife, Spain \\ 13 Ethiopian Space Science and Technology Institute (ESSTI), Entoto Observatory and Research Center (EORC), Astronomy and \\ Astrophysics Research Division, PO Box 33679, Addis Ababa, Ethiopia
}

Received 9 May 2018 / Accepted 20 September 2018

\section{ABSTRACT}

\begin{abstract}
Aims. Our goal is to characterise the dependence of the optical mass-to-light ratio on galaxy colour up to $z=1.5$, expanding the redshift range explored in previous work.

Methods. From the redshifts, stellar masses, and rest-frame luminosities of the ALHAMBRA multi-filter survey, we derive the massto-light ratio versus colour relation for quiescent and for star-forming galaxies. The intrinsic relation and its physical dispersion are derived with a Bayesian inference model.

Results. The rest-frame $i$-band mass-to-light ratio of quiescent and star-forming galaxies presents a tight correlation with the restframe $(g-i)$ colour up to $z=1.5$. The mass-to-light ratio versus colour relation is linear for quiescent galaxies and quadratic for star-forming galaxies. The intrinsic dispersion in these relations is 0.02 dex for quiescent galaxies and 0.06 dex for star-forming ones. The derived relations do not present a significant redshift evolution and are compatible with previous local results in the literature. Finally, these tight relations also hold for $g$ - and $r$-band luminosities.

Conclusions. The derived mass-to-light ratio versus colour relations in ALHAMBRA can be used to predict the mass-to-light ratio from a rest-frame optical colour up to $z=1.5$. These tight correlations do not change with redshift, suggesting that galaxies have evolved along the derived relations during the last $9 \mathrm{Gyr}$.
\end{abstract}

Key words. galaxies: fundamental parameters - galaxies: statistics - galaxies: stellar content

\section{Introduction}

Stellar mass is a fundamental parameter in galaxy evolution studies, presenting correlations with several galaxy properties such as star formation rate (e.g. Noeske et al. 2007; Chang et al. 2015), gasphase metallicity (e.g. Tremonti et al. 2004; Mannucci et al. 2009; Lara-López et al. 2010), stellar content (e.g. Gallazzi et al. 2005, 2014; Díaz-García et al. 2018), galaxy size (e.g. Shen et al. 2003; Trujillo et al. 2007; van der Wel et al. 2014), morphology (e.g.

\footnotetext{
* Based on observations collected at the German-Spanish Astronomical Centre, Calar Alto, jointly operated by the Max-Planck-Institut für Astronomie (MPIA) at Heidelberg and the Instituto de Astrofísica de Andalucía (CSIC).
}

Moffett et al. 2016; Huertas-Company et al. 2016), and nuclear activity (e.g. Kauffmann et al. 2003; Bongiorno et al. 2016).

The measurement of stellar mass in modern photometric and spectroscopic surveys is mainly performed by comparing either an empirical or a theoretical library of templates with the observed spectral energy distributions (SEDs) of galaxies. The mass-to-light ratio associated with the templates, combined with the flux normalisation, provides the stellar mass of a given source (see Courteau et al. 2014 for a recent review on galaxy mass estimation). Thus, understanding and characterising the mass-tolight ratio of different galaxy populations is important in order to derive reliable stellar masses and to minimise systematic differences between data sets and template libraries. 
Mass-to-light ratio versus colour relations (MLCRs) have been studied theoretically and observationally (Tinsley 1981; Jablonka \& Arimoto 1992; Bell \& de Jong 2001; Bell et al. 2003; Portinari et al. 2004; Gallazzi \& Bell 2009; Zibetti et al. 2009; Taylor et al. 2011; Into \& Portinari 2013; McGaugh \& Schombert 2014; Zaritsky et al. 2014; van de Sande et al. 2015; Roediger \& Courteau 2015; Herrmann et al. 2016) in the optical, the ultraviolet (UV), and the near-infrared (NIR). These studies focus on the low-redshift Universe $(z \lesssim 0.5)$ and find welldefined linear MLCRs with low scatter $(<0.2$ dex $)$.

We highlight the work of Taylor et al. (2011, hereafter T11). It is based on SED-fitting to the ugriz broad bands of the Sloan Digital Sky Survey (SDSS DR7, Abazajian et al. 2009) available for the Galaxy And Mass Assembly (GAMA; Driver et al. 2011) survey area. They find a remarkably tight relation $(0.1$ dex dispersion) between the mass-to-light ratio in the $i$ band, denoted $M_{\star} / L_{i}$, and the rest-frame colour $(g-i)$ at $z<0.65$, with a median redshift of $\langle z\rangle=0.2$ for the analysed global population. These authors argue that this small dispersion is driven by (i) the degeneracies of the galaxy templates in such a plane, which are roughly parallel to the MLCR, implying from the theoretical point of view $\sim 0.2$ dex errors in the mass-to-light ratio even with large errors on the derived stellar population parameters; and (ii) the galaxy formation and evolution processes, which are encoded in the observed galaxy colours and only allow a limited set of solutions, making the observed relation even tighter than the theoretical expectations.

In the present work, we expand the results from T11 with the multi-filter Advanced, Large, Homogeneous Area, MediumBand Redshift Astronomical $^{1}$ (ALHAMBRA) survey (Moles et al. 2008). ALHAMBRA provides stellar masses and restframe luminosities thanks to the application of the MUlti-Filter FITing (MUFFIT, Díaz-García et al. 2015) code to 20 optical medium-band and 3 NIR photometric points. In addition, ALHAMBRA covers a wide redshift range, reaching $z=1.5$ with a median redshift of $\langle z\rangle=0.65$, and permits the MLCR of quiescent and star-forming galaxies to be studied separately.

We also refine the statistical estimation of the MLCRs. Instead of performing an error-weighted fit to the data, we applied a Bayesian inference model that accounts for observational uncertainties and includes intrinsic dispersions in the relations (see also Taylor et al. 2015; Montero-Dorta et al. 2016).

The paper is organised as follows. In Sect. 2 we present the ALHAMBRA photometric redshifts, stellar masses, and luminosities. The derived $i$-band MLCRs for quiescent and starforming galaxies and their modelling are described in Sect. 3. Our results are presented and discussed in Sect. 4. The summary and conclusions are in Sect. 5. Throughout this paper we use a standard cosmology with $\Omega_{\mathrm{m}}=0.3, \Omega_{\Lambda}=0.7, \Omega_{\mathrm{k}}=0$, $H_{0}=100 h \mathrm{~km} \mathrm{~s}^{-1} \mathrm{Mpc}^{-1}$, and $h=0.7$. Magnitudes are given in the AB system (Oke \& Gunn 1983). The stellar masses, $M_{\star}$, are expressed in solar masses $\left(M_{\odot}\right)$ and the luminosities, $L$, in units equivalent to an $\mathrm{AB}$ magnitude of 0 . The derived mass-to-light ratios can be transformed into solar luminosities $L_{\odot}$ by subtracting 2.05, 1.90, and 1.81 from the presented MLCRs for the $g$, $r$, and $i$ bands, respectively. With the definitions above, stellar masses can be estimated from the reported mass-to-light ratios as

$\log _{10} M_{\star}=\log _{10}\left(M_{\star} / L\right)-0.4 M$,

where $M$ is the absolute $\mathrm{AB}$ magnitude of the galaxy.

\footnotetext{
WWW . alhambrasurvey. com
}

\section{ALHAMBRA survey}

The ALHAMBRA survey provides a photometric data set over 20 contiguous, equal-width $(\sim 300 \AA)$, non-overlapping, medium-band optical filters $(3500 \AA-9700 \AA)$ plus 3 standard broad-band NIR filters $\left(J, H\right.$, and $\left.K_{\mathrm{s}}\right)$ over eight different regions of the northern sky (Moles et al. 2008). The final survey parameters and scientific goals, as well as the technical properties of the filter set, were described by Moles et al. (2008). The survey collected its data for the 20 plus 3 optical-NIR filters with the $3.5 \mathrm{~m}$ telescope at the Calar Alto observatory, using the widefield camera Large Area Imager for Calar Alto (LAICA) in the optical and the OMEGA-2000 camera in the NIR. The full characterisation, description, and performance of the ALHAMBRA optical photometric system was presented in Aparicio-Villegas et al. (2010). A summary of the optical reduction can be found in Molino et al. (2014), while that of the NIR reduction is in Cristóbal-Hornillos et al. (2009).

\subsection{Bayesian photometric redshifts in ALHAMBRA}

The Bayesian photometric redshifts $\left(z_{\mathrm{b}}\right)$ of ALHAMBRA were estimated with BPZ2, a new version of the Bayesian photometric redshift (BPZ, Benítez 2000) code. The BPZ2 code is a SED-fitting method based in Bayesian inference, where a maximum likelihood is weighted by a prior probability. The main novelties of BPZ2 with respect to its previous version are an updated prior and a new template library that comprises 11 SEDs, with four ellipticals, one lenticular, two spirals, and four starbursts. Further details about BPZ2 can be found in Molino et al. (2014). The ALHAMBRA photometry used to compute the photometric redshifts is PSF-matched, aperture-corrected, and based on isophotal magnitudes (Molino et al. 2014). In addition, a recalibration of the zero point of the images was performed to enhance the accuracy of the photometric redshifts. Sources were detected in a synthetic $F 814 W$ filter image defined to resemble the HST/F814W filter. The total area covered by the current release of the ALHAMBRA survey after masking low signalto-noise areas and bright stars is $2.38 \mathrm{deg}^{2}$ (Arnalte-Mur et al. 2014). The full description of the photometric redshift estimation is detailed in Molino et al. (2014).

The photometric redshift accuracy, as estimated by comparison with spectroscopic redshifts $\left(z_{\mathrm{s}}\right)$, is $\sigma_{\mathrm{NMAD}}=0.012$ at $F 814 W \leq 23$, where $\sigma_{\text {NMAD }}$ is the normalised median absolute deviation of the photometric versus spectroscopic redshift distribution (e.g. Ilbert et al. 2006; Molino et al. 2014). The fraction of catastrophic outliers with $\left|z_{\mathrm{b}}-z_{\mathrm{s}}\right| /\left(1+z_{\mathrm{s}}\right)>0.2$ is $2.1 \%$. We refer the reader to Molino et al. (2014) for a more detailed discussion.

\subsection{MUFFIT: stellar masses and rest-frame colours}

The BPZ2 template library discussed above is empirical, and the different templates do not have mass-to-light ratios assigned a priori. Hence, an alternative methodology is needed to compute the stellar mass of the ALHAMBRA sources.

The MUFFIT code is specifically performed and optimised to deal with multi-photometric data, such as the ALHAMBRA data set, through the SED-fitting (based in a $\chi^{2}$-test weighted by errors) to mixtures of two simple stellar populations (SSPs; a dominant "old" component plus a posterior star formation episode, which can be related with a burst or a younger/extended tail in the star formation history). MUFFIT includes an iterative process for removing those bands that may be affected by strong 


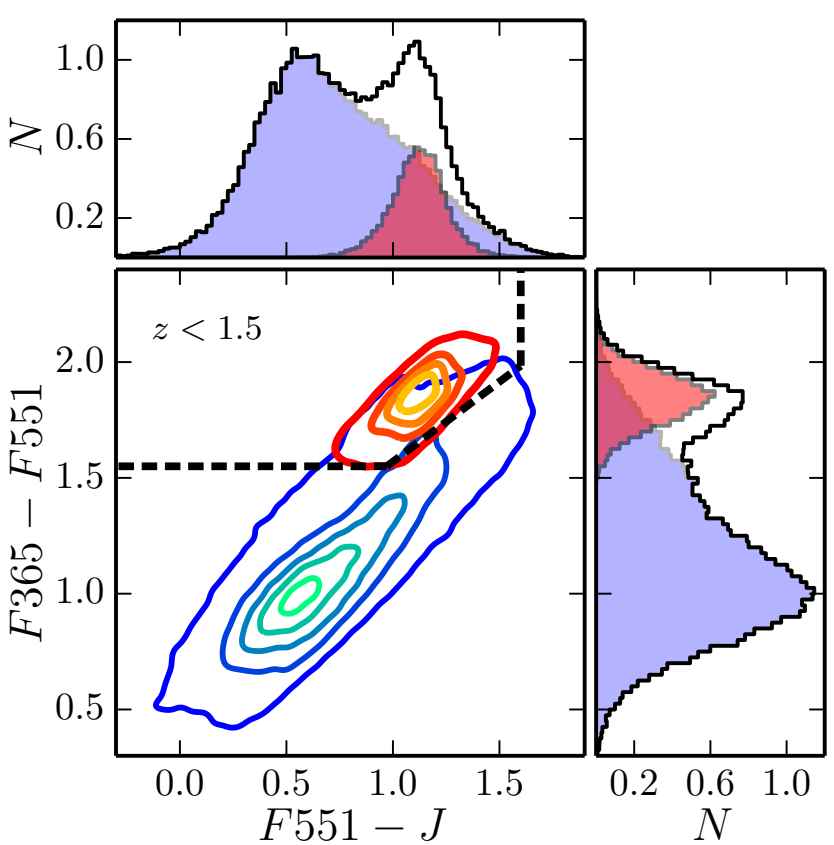

Fig. 1. Rest-frame colour-colour plane $F 365-F 551$ versus $F 551-J$ for the 76642 ALHAMBRA galaxies with $F 814 W \leq 23$ at $z<1.5$. This plane is equivalent to the commonly used $U V J$ diagram, and the quiescent selection box from the literature (Williams et al. 2009) is delimited by dashed lines. The coloured lines show level contours in the density of galaxies, starting at 0.1 galaxies $\mathrm{dex}^{-2}$ and increasing in 0.6 galaxies dex ${ }^{-2}$ steps. Red contours show the quiescent population, and blue contours show the star-forming population, as defined by DG17. The side panels show the normalised distribution in F365 - F551 (right panel) and $F 551-J$ (top panel). In both panels the total distribution is presented in black, the quiescent population in filled red, and the starforming population in filled blue.

emission lines so as to carry out a detailed analysis of the galaxy SEDs even when strong nebular or active galactic nuclei (AGN) emission lines are present, which may be especially troublesome for intermediate- and narrow-band surveys. The two SSPs involved in the modelling share the same initial mass function (IMF) and extinction, but have independent ages and metallicities. The final number of free parameters in the fitting is eight (two ages, two metallicities, one extinction, the total stellar mass, the mass fraction in the older component, and the redshift), with a fixed IMF. ALHAMBRA sources with $F 814 W \leq 23$ are analysed with MUFFIT by Díaz-García et al. (2017, hereafter DG17), retrieving ages, metallicities, stellar masses, rest-frame luminosities, and extinctions. MUFFIT also provides photometric redshifts, using the BPZ2 solutions presented in the previous section as a prior to minimise degeneracies. This improves the photometric redshift accuracy by $\sim 20 \%$ and decreases the fraction of outliers by $\sim 10 \%$ (DG17). The retrieved parameters are in good agreement with both spectroscopic diagnostics from SDSS data and photometric studies in the COSMOS survey, where the galaxy samples overlap (Díaz-García et al. 2015, DG17).

To study the MLCR of ALHAMBRA galaxies and its redshift evolution, we used the redshifts, stellar masses, and rest-frame luminosities in the gri broad bands derived by MUFFIT. These parameters were estimated assuming Bruzual \& Charlot (2003, BC03) stellar population models, Fitzpatrick (1999) extinction law, and Chabrier (2003) IMF. We refer the reader to Díaz-García et al. $(2015,2018)$, and DG1 7 for further details about MUFFIT and the quantities derived.

\subsection{Selection of quiescent and star-forming galaxies}

Throughout this paper, we focus our analysis on the galaxies in the ALHAMBRA gold catalogue ${ }^{2}$. This catalogue comprises $\sim 100 \mathrm{k}$ sources with $F 814 W \leq 23$ (Molino et al. 2014).

We split our galaxies into quiescent and star-forming with the dust-corrected version of the $U V J$ colour-colour plane presented in DG17, adapted to the ALHAMBRA medium-band filter system: we used $F 365$ instead of the filter $U$ and $F 551$ instead of the filter $V$; the ALHAMBRA filter $J$ is the standard one. As shown by DG17, quiescent and star-forming galaxies with $F 814 W \leq 23$ define two non-overlapping populations in the colour-colour plane after removing dust effects, with the selection boundary located at $(F 365-F 551)=1.5$. We refer the reader to DG17 for a detailed description of the selection process and the study of the stellar population properties of quiescent galaxies in the $U V J$ colour-colour plane. We show the observed (i.e. reddened by dust) rest-frame distribution of the 76642 ALHAMBRA gold catalogue galaxies with $z<1.5$ in Fig. 1. The quiescent population is enclosed by the common colour-colour selection box (Williams et al. 2009), but a population of dusty star-forming galaxies is also located in this area. DG17 show that a significant fraction $(\sim 20 \%)$ of these red galaxies are indeed dusty and star-forming, and are contaminating the quiescent population. Thanks to the low-resolution spectral information from ALHAMBRA, the MUFFIT code is able to provide a robust quiescent versus star-forming classification.

The final sample, having $z<1.5$ and $F 814 W \leq 23$, comprises 12905 quiescent and 63737 star-forming galaxies. The stellar masses covered by our data span the $8<\log _{10} M_{\star} / M_{\odot}<$ 11.5 range. Further details about the stellar mass completeness and the redshift distribution of the sample are presented in DG17. We study the MLCR of these samples in the next section.

\section{Mass-to-light ratio versus colour relation at $z<1.5$}

\subsection{Observed relation for quiescent and star-forming galaxies}

In this section, we study the relation between the mass-to-light ratio in the $i$-band and the observed rest-frame $(g-i)$ colour of the ALHAMBRA galaxies with $z<1.5$. In some cases, we denote $\Upsilon=\log _{10}\left(M_{\star} / L_{i}\right)$ and $C=(g-i)$ for the sake of clarity. The redshift, stellar masses, and observed rest-frame (i.e. reddened by dust) luminosities were derived by the MUFFIT code (Sect. 2.2).

We present the $M_{\star} / L_{i}$ versus $(g-i)$ colour plane for both quiescent and star-forming galaxies in the top panel of Fig. 2. We find that for both populations, the mass-to-light ratio increases for redder colours, in agreement with the literature (see references in Sect. 1). We describe the modelling of this dependence in Sect. 3.2. In the figure, we also present the MLCR found by T11 in the GAMA survey. Their relation is in excellent agreement with our observed values: the comparison between our measurements and their predictions, $\Delta \Upsilon=\Upsilon-\Upsilon_{\mathrm{T} 11}(C)$, has no bias, $\langle\Delta \Upsilon\rangle=0.01 \mathrm{dex}$, and a small dispersion of $\sigma_{\Delta \Upsilon}=0.1 \mathrm{dex}$ (bottom panel in Fig. 2). We note that T11 use BC03 stellar population models and a Chabrier (2003) IMF, as we did, but different extinction laws (cf. Calzetti et al. 2000 and Fitzpatrick 1999) and star formation histories (SFHs; $e$-fold tau models versus two stellar populations mix) were assumed.

\footnotetext{
2 http://cosmo.iaa.es/content/ALHAMBRA-Gold-catalog
} 

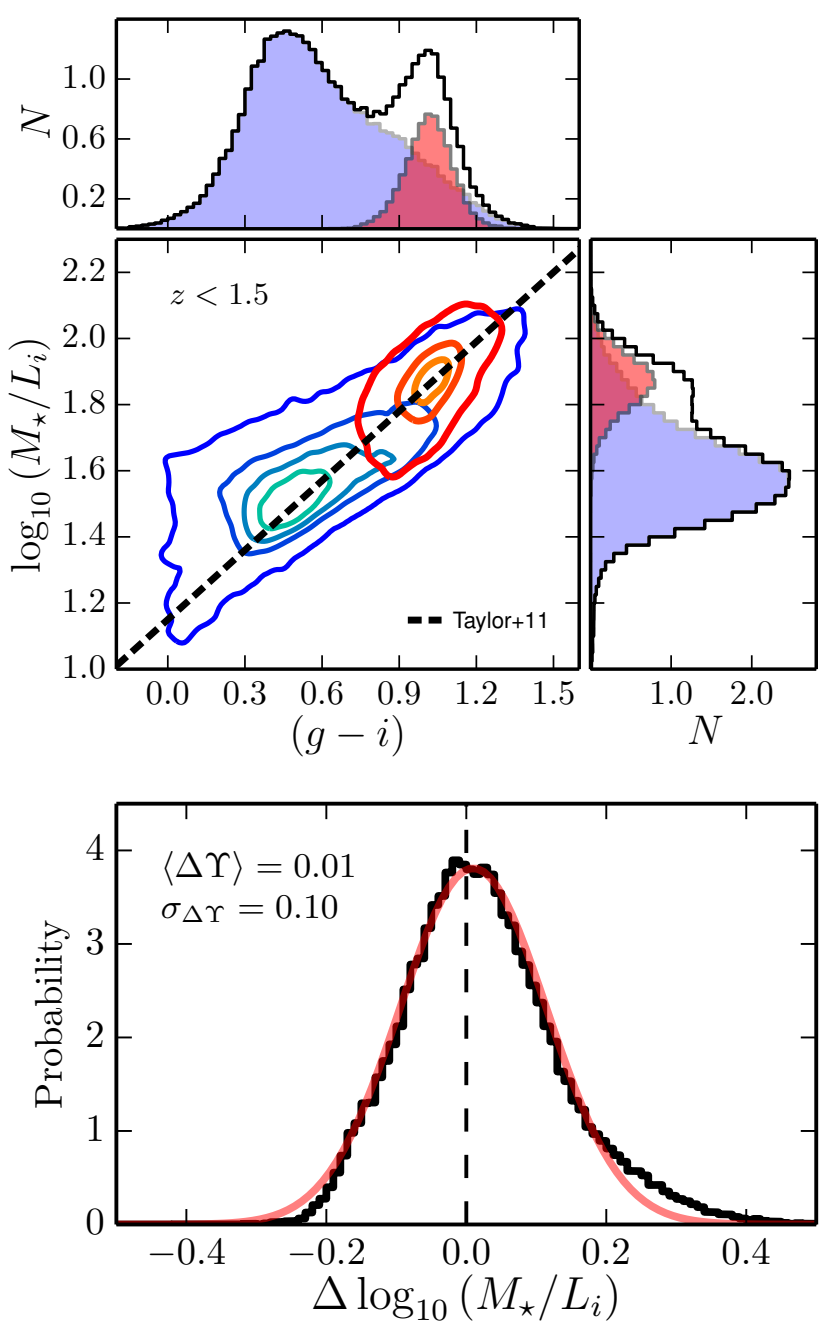

Fig. 2. Top panel: mass-to-light ratio $M_{\star} / L_{i}$ as a function of the restframe colour $g-i$ for the 76642 ALHAMBRA galaxies with $F 814 W \leq$ 23 at $z<1.5$. The coloured lines show level contours in the density of galaxies, starting at 0.1 galaxies $\mathrm{dex}^{-2}$ and increasing in 1.5 galaxies $\operatorname{dex}^{-2}$ steps. Red contours show the quiescent population, and blue contours the star-forming one. The side panels show the normalised distribution in $g-i$ (upper panel) and $\log _{10}\left(M_{\star} / L_{i}\right)$ (right panel). In both panels the total distribution is presented in black, the quiescent population in filled red, and the star-forming population in filled blue. The black dashed line marks the relation derived by Taylor et al. (2011) at $z<0.65$ using GAMA galaxies and SDSS five-band photometry. Bottom panel: comparison between the observed ALHAMBRA massto-light ratio and that expected from the Taylor et al. (2011) relation. The red solid line is the best Gaussian fit with median $\langle\Delta \Upsilon\rangle=0.01 \mathrm{dex}$ and dispersion $\sigma_{\Delta \Upsilon}=0.10$ dex.

The T11 study is performed using a sample of $z<0.65$ galaxies with a median redshift of $\langle z\rangle=0.2$, and our data covers a wider redshift range $(z \leq 1.5)$ with a median redshift of $\langle z\rangle=0.65$. This suggests that the low-redshift relation measured by T11 in GAMA has not evolved significantly with redshift. We assume this redshift independence in the following and test it in Sect. 4.2.

\subsection{Modelling the intrinsic mass-to-light versus colour relation}

The measurements presented in the previous section are affected by observational errors, blurring the information and biasing our analysis. We are interested in the intrinsic distribution of our measurements in the mass-to-light ratio versus colour plane, and in this section we detail the steps to estimate it. The results are presented in Sect. 4.

The intrinsic distribution of interest is noted by $D$, and provides the real values of our measurements for a set of parameters $\theta$

$D\left(\Upsilon_{0}, C_{0} \mid \theta\right)$

where $\Upsilon_{0}$ and $C_{0}$ are the real values of the mass-to-light ratio and the colour unaffected by observational errors. We derive the posterior of the parameters $\theta$ that define the intrinsic distribution $D$ for both quiescent and star-forming galaxies with a Bayesian model. Formally,

$P\left(\theta \mid \Upsilon, C, \sigma_{\Upsilon}, \sigma_{C}\right) \propto \mathcal{L}\left(\Upsilon, C \mid \theta, \sigma_{\Upsilon}, \sigma_{C}\right) P(\theta)$,

where $\sigma_{\Upsilon}$ and $\sigma_{C}$ are the uncertainties in the observed mass-tolight ratio and $(g-i)$ colour, respectively, $\mathcal{L}$ is the likelihood of the data given $\theta$, and $P(\theta)$ the prior in the parameters. The posterior probability is normalised to one.

The likelihood function associated with our problem is

$\mathcal{L}\left(\Upsilon, C \mid \theta, \sigma_{\Upsilon}, \sigma_{C}\right)=\prod_{k} P_{k}\left(\Upsilon_{k}, C_{k} \mid \theta, \sigma_{\Upsilon, k}, \sigma_{C, k}\right)$,

where the index $k$ spans the galaxies in the sample, and $P_{k}$ traces the probability of the measurement $k$ for a set of parameters $\theta$. This probability can be expressed as

$$
\begin{aligned}
& P_{k}\left(\Upsilon_{k}, C_{k} \mid \theta, \sigma_{\Upsilon, k}, \sigma_{C, k}\right)= \\
& \quad \int D\left(\Upsilon_{0}, C_{0} \mid \theta\right) P_{\mathrm{G}}\left(\Upsilon_{k} \mid \Upsilon_{0}, \sigma_{\Upsilon, k}\right) P_{\mathrm{G}}\left(C_{k} \mid C_{0}, \sigma_{C, k}\right) \mathrm{d} \Upsilon_{0} \mathrm{~d} C_{0},
\end{aligned}
$$

where the real values $\Upsilon_{0}$ and $C_{0}$ derived from the model $D$ are affected by Gaussian observational errors,

$$
P_{\mathrm{G}}\left(x \mid x_{0}, \sigma_{x}\right)=\frac{1}{\sqrt{2 \pi} \sigma} \exp \left[-\frac{\left(x-x_{0}\right)^{2}}{2 \sigma^{2}}\right],
$$

providing the likelihood of an observed value given its real value and uncertainty. We have no access to the real values $\Upsilon_{0}$ and $C_{0}$, so we marginalised over them in Eq. (5) and the likelihood is expressed therefore with known quantities; the marginalisation ranges were $C_{0} \in[-0.5,2.5]$ and $\Upsilon_{0} \in[0.5,3.0]$. We assumed no covariance between $\Upsilon$ and $C$, although they share the $i$-band luminosity information. We checked by Monte Carlo sampling of the $M_{\star}, L_{i}$, and $L_{g}$ distributions that the covariance is small, with $\rho_{\Upsilon \mathrm{C}} \sim 0.05$. Hence, we disregard the covariance term for simplicity.

We explore the parameters posterior distribution with the emcee code (Foreman-Mackey et al. 2013), a Python implementation of the affine-invariant ensemble sampler for Markov chain Monte Carlo (MCMC) proposed by Goodman \& Weare (2010). The emcee code provides a collection of solutions in the parameter space, denoted $\theta_{\mathrm{MC}}$, with the density of solutions being proportional to the posterior probability of the parameters. We obtained central values of the parameters as the median, denoted $\left\langle\theta_{\mathrm{MC}}\right\rangle$, and their uncertainties as the range enclosing $68 \%$ of the projected solutions around the median.

We define in the following the distributions assumed for the quiescent and star-forming populations, and the prior imposed to their parameters. The quiescent $(\mathrm{Q})$ population is described as

$D_{\mathrm{Q}}\left(\Upsilon_{0}, C_{0} \mid \theta_{\mathrm{Q}}\right)=P_{\mathrm{G}}\left(C_{0} \mid \mu_{\mathrm{Q}}, s_{\mathrm{Q}}\right) P_{\mathrm{G}}\left(\Upsilon_{0} \mid A_{\mathrm{Q}}+B_{\mathrm{Q}} C_{0}, \sigma_{\mathrm{Q}}\right)$, 
where $\mu_{\mathrm{Q}}$ and $s_{\mathrm{Q}}$ describe the intrinsic Gaussian $(g-i)$ colour distribution, $A_{\mathrm{Q}}$ and $B_{\mathrm{Q}}$ are the coefficients that define the MLCR, and $\sigma_{\mathrm{Q}}$ the intrinsic (i.e. related to physical processes) dispersion of the relation. We have a set of five parameters to describe the distribution of quiescent galaxies: $\theta_{\mathrm{Q}}=\left\{\mu_{\mathrm{Q}}, s_{\mathrm{Q}}, A_{\mathrm{Q}}, B_{\mathrm{Q}}, \sigma_{\mathrm{Q}}\right\}$. We used flat priors, $P\left(\theta_{\mathrm{Q}}\right)=1$, except for the dispersions $s_{\mathrm{Q}}$ and $\sigma_{\mathrm{Q}}$, that we imposed as positive.

The star-forming (SF) population presents a more complex behaviour (Fig. 2), and we modelled it as

$$
\begin{aligned}
D_{\mathrm{SF}}\left(\Upsilon_{0}, C_{0} \mid \theta_{\mathrm{SF}}\right)= & P_{\mathrm{G}}\left(C_{0} \mid \mu_{\mathrm{SF}}, s_{\mathrm{SF}}\right)\left[1+\operatorname{erf}\left(\alpha_{\mathrm{SF}} \frac{C_{0}-\mu_{\mathrm{SF}}}{\sqrt{2} s_{\mathrm{SF}}}\right)\right] \\
& P_{\mathrm{G}}\left(\Upsilon_{0} \mid A_{\mathrm{SF}}+B_{\mathrm{SF}} C_{0}+C_{\mathrm{SF}} C_{0}^{2}, \sigma_{\mathrm{SF}}\right)
\end{aligned}
$$

where $\mu_{\mathrm{SF}}, s_{\mathrm{SF}}$, and $\alpha_{\mathrm{SF}}$ describe the intrinsic $(g-i)$ colour distribution; $A_{\mathrm{SF}}, B_{\mathrm{SF}}$, and $C_{\mathrm{SF}}$ are the coefficients that define the MLCR for star-forming galaxies; and $\sigma_{\mathrm{SF}}$ the intrinsic dispersion of the relation. Important differences are present compared with the quiescent population. The distribution of $C_{0}$ is not symmetric (Fig. 2), and we accounted for this asymmetry by adding the error function term and the parameter $\alpha_{\mathrm{SF}}$, which controls the skewness of the distribution (Azzalini 2005). In addition, a second-order polynomial is preferred over a first-order one to describe the dependence of $\Upsilon_{0}$ on colour. This is motivated by the apparent curvature present at the redder colours in Fig. 2. To choose between the linear or the parabolic MLCR, we used the Bayesian information criterion (BIC, Schwarz 1978), defined as

$\mathrm{BIC}=N \log n-2 \log \mathcal{L}\left(\Upsilon, C \mid\left\langle\theta_{\mathrm{MC}}\right\rangle\right)$,

where $N$ is the number of parameters in the model and $n$ the number of galaxies in the sample. We find $\triangle \mathrm{BIC}=\mathrm{BIC}_{\mathrm{par}}-\mathrm{BIC}_{\text {lin }}=$ -750 , favouring the inclusion of $C_{\mathrm{SF}}$ in the modelling. For consistency, we checked the application of a parabolic MLCR for quiescent galaxies. We found $\triangle \mathrm{BIC}=\mathrm{BIC}_{\mathrm{par}}-\mathrm{BIC}_{\text {lin }}=1.5$, thus favouring the simpler linear model. Figure 2 also suggests an asymmetric distribution in $\Upsilon_{0}$, instead of the assumed Gaussian. We also studied the inclusion of an additional skew parameter for $\Upsilon_{0}$, but it was consistent with zero and in this case the BIC favours the simpler Gaussian model without the extra skew parameter. Thus, we have a set of seven parameters to describe the distribution of star-forming galaxies, $\theta_{\mathrm{SF}}=$ $\left\{\mu_{\mathrm{SF}}, s_{\mathrm{SF}}, \alpha_{\mathrm{SF}}, A_{\mathrm{SF}}, B_{\mathrm{SF}}, C_{\mathrm{SF}}, \sigma_{\mathrm{SF}}\right\}$. We used flat priors, $P\left(\theta_{\mathrm{SF}}\right)=$ 1 , except for the dispersions $s_{\mathrm{SF}}$ and $\sigma_{\mathrm{SF}}$, which we imposed as positive.

We note that the redshift dimension is not included in our analysis because we are assuming that the MLCRs do not depend on redshift. This was initially motivated by the excellent agreement with the local relation from T11 shown in Fig. 2, and we further test this assumption in Sect. 4.2.

\section{Results}

We present the derived $i$-band MLCRs for both quiescent and star-forming galaxies in Sect. 4.1, and explore the redshift dependence of the relations in Sect. 4.2. The $g$ - and $r$-band MLCRs are presented in Sect. 4.3, and we compare our results with the literature in Sect. 4.4.

\subsection{Mass-to-light ratio versus colour relation for quiescent and star-forming galaxies}

In this section, we present the results of our modelling. They are summarised in Fig. 3 for quiescent galaxies and in Fig. 4
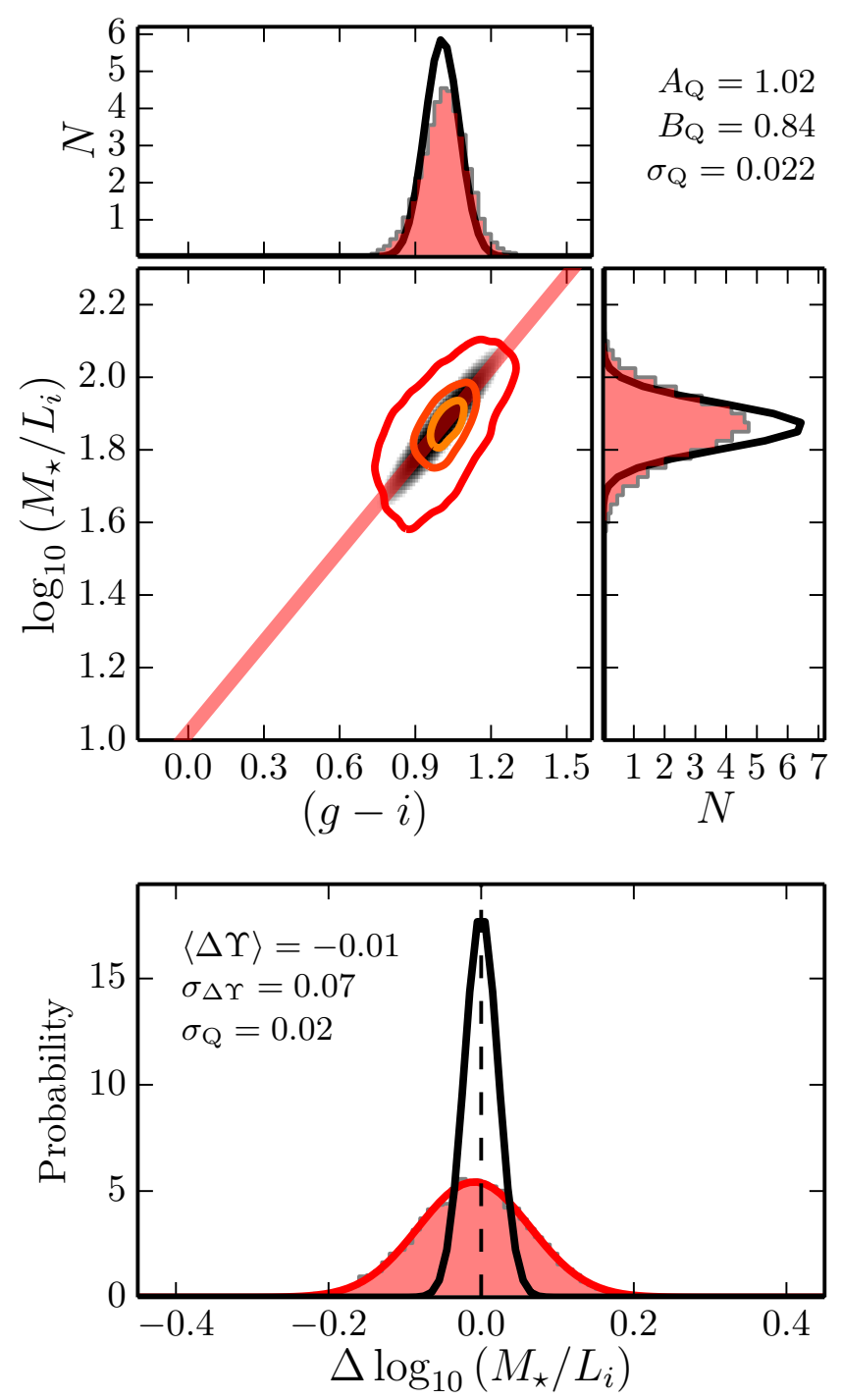

Fig. 3. Top panel: mass-to-light ratio $M_{\star} / L_{i}$ as a function of the restframe colour $g-i$ for the 12905 ALHAMBRA quiescent galaxies with $F 814 W \leq 23$ at $z<1.5$. The solid lines show level contours in the density of galaxies as in Fig. 2. The grey scale shows the median fitting model to the data, $D_{\mathrm{Q}}\left(\Upsilon_{0}, C_{0} \mid\left\langle\theta_{\mathrm{Q}}\right\rangle\right)$. The red area represents the derived MLCR, $\log _{10}\left(M_{\star} / L_{i}\right)=1.02+0.84(g-i)$, and its $1 \sigma$ intrinsic dispersion, $\sigma_{\mathrm{Q}}=0.02$. Side panels: normalised projected histogram in $g-i$ (upper panel) and $\log _{10}\left(M_{\star} / L_{i}\right)$ (right panel). In both panels the observed distribution is presented in filled red, and the derived median model in solid black. Bottom panel: comparison between the observed ALHAMBRA mass-to-light ratio and that expected from our median relation (red filled histogram). The solid red line is the best Gaussian fit with median $\langle\Delta \Upsilon\rangle=-0.01$ and $\sigma_{\Delta \Upsilon}=0.07$. The solid black line illustrates the estimated intrinsic dispersion unaffected by observational uncertainties.

for the star-forming galaxies. The derived parameters are compiled in Table 1 . In both cases we find that the assumed model satisfactorily describes the observed distributions in colour and mass-to-light ratio spaces.

We start by presenting the results for quiescent galaxies. We estimate

$\Upsilon_{\mathrm{Q}}=1.02+0.84(g-i)$

with a small intrinsic dispersion of $\sigma_{\mathrm{Q}}=0.02 \mathrm{dex}$. The observed dispersion, which includes the observational errors, was estimated from a Gaussian fit to the distribution of the variable 

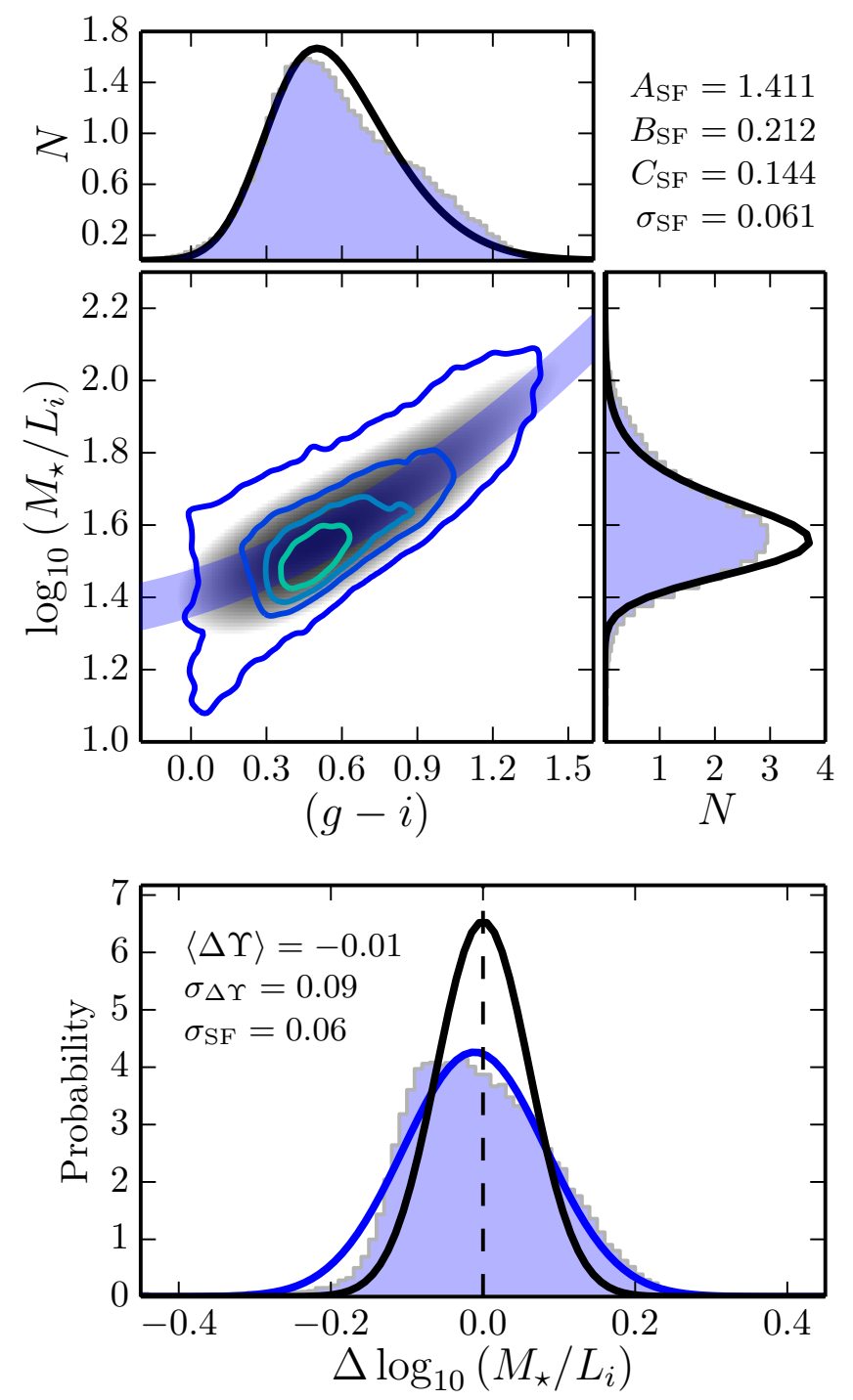

Fig. 4. Top panel: mass-to-light ratio $M_{\star} / L_{i}$ as a function of the restframe colour $g-i$ for the 63737 ALHAMBRA star-forming galaxies with $F 814 W \leq 23$ at $z<1.5$. The solid lines show level contours in the density of galaxies as in Fig. 2. The grey scale shows the median fitting model to the data, $D_{\mathrm{SF}}\left(\Upsilon_{0}, C_{0} \mid\left\langle\theta_{\mathrm{SF}}\right\rangle\right)$. The blue area represents the derived MLCR, $\log _{10}\left(M_{\star} / L_{i}\right)=1.411+0.212(g-i)+0.144(g-i)^{2}$, and its $1 \sigma$ intrinsic dispersion, $\sigma_{\mathrm{SF}}=0.06$. Side panels: normalised projected histogram in $g-i$ (upper panel) and $\log _{10}\left(M_{\star} / L_{i}\right)$ (right panel). In both panels the observed distribution is presented in filled blue, and the derived median model in solid black. Bottom panel: comparison between the observed ALHAMBRA mass-to-light ratio and that expected from our median relation (blue filled histogram). The blue solid line is the best Gaussian fit with median $\langle\Delta \Upsilon\rangle=-0.01$ and $\sigma_{\Delta \Upsilon}=0.09$. The black solid line illustrates the estimated intrinsic dispersion unaffected by observational uncertainties.

$\Delta \Upsilon=\Upsilon-\Upsilon_{\mathrm{Q}}$, yielding $\sigma_{\Delta \Upsilon}=0.07$ dex (bottom panel in Fig. 3). This value is lower than the 0.1 dex obtained with the local MLCR from T11.

For star-forming galaxies, we find

$\Upsilon_{\mathrm{SF}}=1.411+0.212(g-i)+0.144(g-i)^{2}$

with an intrinsic dispersion of $\sigma_{\mathrm{SF}}=0.06$ dex. The observed dispersion in this case is $\sigma_{\Delta \Upsilon}=0.09 \mathrm{dex}$ (bottom panel in Fig. 4), similar to the 0.1 dex obtained with the T11 relation. The higher complexity of the star-forming population is not surprising, due to the combination of an underlying old population that dominates the stellar mass, a young population dominating the emission in the bluer bands, and the presence of different dust content. Even so, a well-defined MLCR with a small dispersion is inferred from our data.

We conclude that the encouraging 0.1 dex precision in the mass-to-light ratio estimation from the optical colour $(g-i)$ found by T11 is even tighter after the observational uncertainties are accounted for. The dispersion derived with ALHAMBRA data at $z<1.5$ is 0.02 dex for quiescent galaxies and 0.06 dex for star-forming galaxies. These small dispersions refer to the statistical analysis of the data, and systematic uncertainties related with the assumed stellar population models, IMF, SFHs, extinction law, etc., are not included in the analysis (see Portinari et al. 2004; Conroy et al. 2009; Barro et al. 2011, and Courteau et al. 2014 , for a detailed discussion about systematics in stellar mass estimations). The similarity between the T11 values and ours suggests that the assumed extinction law and the SFHs are not an important source of systematics, with stellar population models and the IMF being the main contributors. The application of different stellar population models, such as those from Vazdekis et al. (2016), Maraston (2005), or Conroy \& Gunn (2010), is beyond the scope of the present work.

\subsection{Redshift evolution of the mass-to-light ratio versus colour relation}

The results presented in the previous section imply a tight relation of the mass-to-light ratio with the optical colour $(g-i)$. In our analysis, we assumed such a relation as redshift independent, motivated by the nice agreement with the $z \sim 0.2$ results from T11 (Fig. 2).

We present the redshift evolution of $\Delta \Upsilon$ in Fig. 5, both for quiescent and star-forming galaxies. We found no evidence of redshift evolution for either galaxy population, with the median $|\Delta \Upsilon|$ at any redshift always below 0.02 dex. We performed a linear fit to constrain the possible residual evolution of $\Upsilon$ with redshift, finding $\Delta \Upsilon_{\mathrm{Q}} \propto 0.04 z$ for quiescent galaxies and $\Delta \Upsilon_{\mathrm{SF}} \propto$ $0.01 z$ for star-forming galaxies. We conclude therefore that the relations presented in Eqs. (10) and (11) have not changed appreciably during the last 9 Gyr of the Universe, with quiescent and star-forming galaxies evolving along the derived relations since $z=1.5$.

\subsection{Mass-to-light ratio versus colour relation in the $r$ and $g$ bands}

We complement the results in the previous sections with the estimation of the intrinsic relation between the mass-to-light ratio in the $r$ and $g$ bands with $(g-i)$ colour, both for quiescent and starforming galaxies. We confirm the tight relations found with the $i$-band luminosity and the curvature for the star-forming population. We present the estimated relations in Table 1 for future reference.

We find that the normalisation of the MLCRs is similar in the gri bands at the 0.05 dex level. This occurs because our luminosities are expressed in $\mathrm{AB}$ units, so a null colour implies the same luminosity in all the bands which share a common stellar mass.

Regarding the slope for the quiescent population, it is larger for bluer bands. This implies that at the median colour of the quiescent population, $\langle(g-i)\rangle=1$, the mass-to-light ratio decreases from $\log _{10}\left(M_{\star} / L_{g}\right)=2.26$ to $\log _{10}\left(M_{\star} / L_{i}\right)=1.86$, reflecting the larger contribution to the stellar mass budget of redder lowmass stars. 
Table 1. ALHAMBRA mass-to-light ratio versus $(g-i)$ colour relation.

\begin{tabular}{lccccccc}
\hline \hline Optical band & Galaxy type & $A$ & $B$ & $C$ & $\sigma_{\text {int }}$ & $\sigma_{\Delta \mathrm{Y}}$ & $\Delta \mathrm{BIC}$ \\
\hline \multirow{2}{*}{ band } & Quiescent & $1.02 \pm 0.01$ & $0.84 \pm 0.01$ & $\ldots$ & $0.022 \pm 0.001$ & 0.07 & 1.5 \\
& Star-forming & $1.411 \pm 0.003$ & $0.212 \pm 0.007$ & $0.144 \pm 0.005$ & $0.061 \pm 0.001$ & 0.09 & -750 \\
$r$ band & Quiescent & $1.02 \pm 0.01$ & $1.01 \pm 0.01$ & $\ldots$ & $0.021 \pm 0.001$ & 0.08 & 5.5 \\
& Star-forming & $1.453 \pm 0.003$ & $0.373 \pm 0.008$ & $0.128 \pm 0.007$ & $0.063 \pm 0.001$ & 0.10 & -510 \\
$g$ band & Quiescent & $0.98 \pm 0.02$ & $1.28 \pm 0.02$ & $\ldots$ & $0.014 \pm 0.001$ & 0.07 & 1.5 \\
& Star-forming & $1.386 \pm 0.003$ & $0.707 \pm 0.009$ & $0.078 \pm 0.007$ & $0.057 \pm 0.001$ & 0.09 & -190 \\
\hline
\end{tabular}
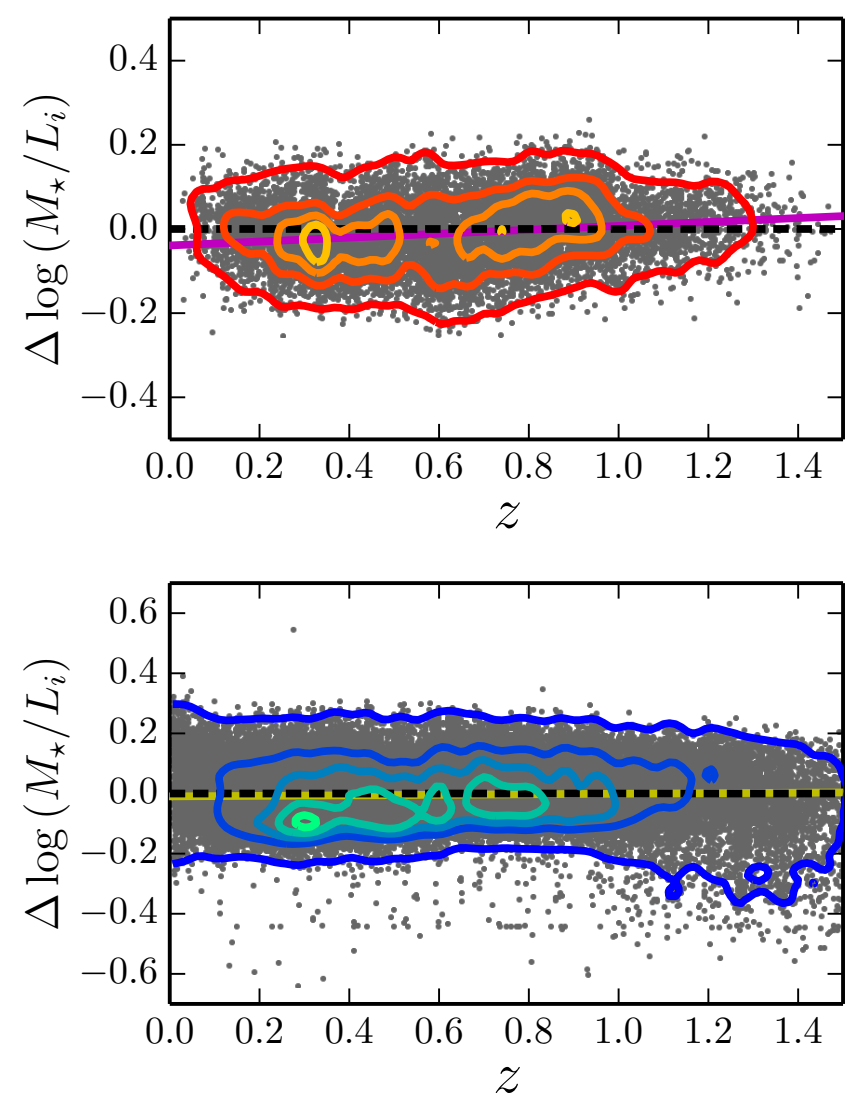

Fig. 5. Comparison between the observed ALHAMBRA mass-to-light ratio and the expected ratio from our median relation as a function of redshift for quiescent (top panel) and star-forming (bottom panel) galaxies with $F 814 W \leq 23$ (grey dots). The solid contours show levels in the number of galaxies, starting at one galaxy and increasing in steps of five galaxies for quiescent galaxies, and in steps of 15 galaxies for star-forming galaxies. The dashed lines mark null difference. The solid lines show the linear fit to the mass-to-light ratio residuals, $\Delta \Upsilon_{\mathrm{Q}} \propto 0.04 z$ (top panel) and $\Delta \Upsilon_{\mathrm{SF}} \propto 0.01 z$ (bottom panel).

In the case of the star-forming galaxies, the parameter $B_{\mathrm{SF}}$ is larger at bluer bands, but the parameter $C_{\mathrm{SF}}$ is smaller. This implies a lower curvature of the MLCR in the $g$ band. We checked that the quadratic model is still favoured by the data even in the $g$-band case, with $\Delta \mathrm{BIC}=-190$ (Table 1).

The intrinsic dispersion in the MLCRs is still low and similar to the $i$-band values, with $\sigma_{\mathrm{Q}} \sim 0.02$ dex and $\sigma_{\mathrm{SF}} \sim$ 0.06 dex. Finally, the observed dispersion, affected by observational errors, are also similar to the fiducial $i$-band values, as summarised in Table 1.

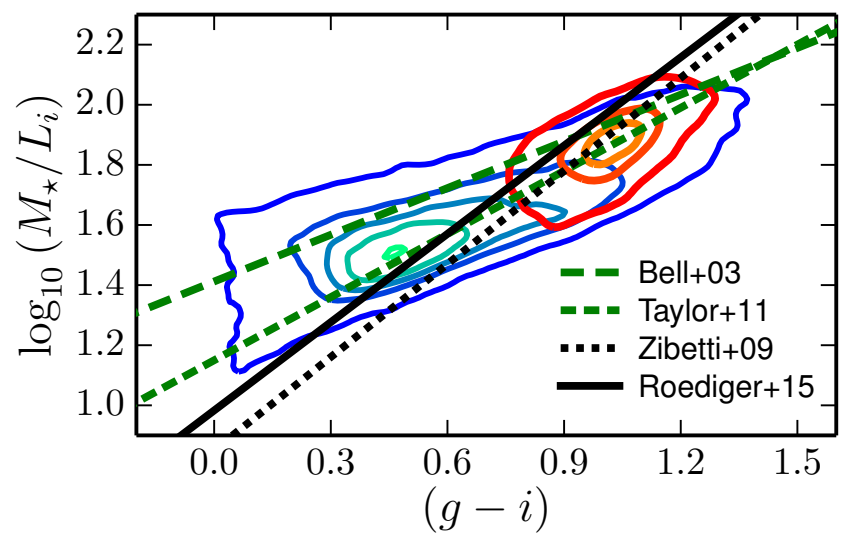

Fig. 6. Comparison of the observed mass-to-light ratio versus $(g-i)$ colour in ALHAMBRA with MLCRs from the literature. The contours are the same as in Fig. 2. The dashed green line is from the observational study of T11. The other black lines are from theoretical expectations: Zibetti et al. (2009, dotted) and Roediger \& Courteau (2015, solid). All the MLCRs have been scaled to a Chabrier (2003) IMF and referred to BC03 models.

We conclude that the MLCR holds in the optical range covered by the gri bands, confirming the tight correlation between optical mass-to-light ratios and the rest-frame colour $(g-i)$.

\subsection{Comparison with the literature}

In addition to the T11 work, several studies in the literature have tackled the problem of the MLCR, both theoretically and observationally (see references in Sect. 1). We present the $i$-band mass-to-light ratio versus $(g-i)$ colour from previous work in Fig. 6 . We only present the colour range imposed by the ALHAMBRA data, $0<(g-i)<1.5$ (Fig. 2). All the MLCRs have been scaled to a Chabrier (2003) IMF and referred to BC03 stellar population models to minimise systematic differences.

We find a reasonably good agreement with the theoretical results from Roediger \& Courteau (2015) and Zibetti et al. (2009). The comparison of these predictions with our values yields a bias of $\langle\Delta \Upsilon\rangle=-0.01$ and 0.08 , and a dispersion of $\sigma_{\Delta \Upsilon}=0.17$ and 0.19 , respectively. We highlight the predictions from Roediger \& Courteau (2015), which have no bias and a dispersion only a factor of two larger than our optimal MLCRs.

From the observational point of view, we recall the agreement with the results from T11 (Fig. 2). Their relation provides no bias and a dispersion of $\sigma_{\Delta \Upsilon}=0.1$. We also compare our results with the popular work by Bell et al. (2003). Their relation yields a bias of $\langle\Delta \Upsilon\rangle=-0.12$ and again a dispersion of $\sigma_{\Delta \Upsilon}=0.1$. We note that the MLCR of Bell et al. (2003) was estimated with the PEGASE (Fioc \& Rocca-Volmerange 1997) 
stellar populations models and a "diet Salpeter" IMF. Hence, we applied a -0.10 dex offset to the relations in Bell et al. (2003) to account for the difference in the stellar population models ${ }^{3}$, and $\mathrm{a}-0.15$ dex offset to scale the IMF.

Following T11, we conclude that the range of colours covered by the observed galaxies, which are a consequence of their formation and evolution, restrict the parameter space of the models and provide tighter MLCRs than expected from theory. The bias with respect to previous work is at the $\sim 0.1$ dex level, supporting the tight relations derived from ALHAMBRA data.

\section{Summary and conclusions}

We used the redshifts, stellar masses and rest-frame colours derived with MUFFIT for 76642 ALHAMBRA sources at $z \leq 1.5$ to explore the $i$-band mass-to-light ratio relation with the restframe $(g-i)$ colour. As shown by T11, there is a tight $(0.1 \mathrm{dex})$ MLCR in the GAMA survey at $z \sim 0.2$, and we expand their study up to $z=1.5$.

We found that the $i$-band MLCR is also present in ALHAMBRA at $z \leq 1.5$, for both quiescent and star-forming galaxies. The data suggests a linear MLCR for quiescent galaxies and a quadratic one for star-forming systems, as summarised in Table 1, and also holds for $g$ and $r$ luminosities.

The derived relations present an intrinsic dispersion, after accounting for observational uncertainties, of $\sigma_{\mathrm{Q}}=0.02 \mathrm{dex}$ and $\sigma_{\mathrm{SF}}=0.06 \mathrm{dex}$. These dispersions are intrinsic and must be accounted for in addition to the colour error when deriving the mass-to-light ratio uncertainty. We also note that they refer to statistical dispersions, and the final error budget in massto-light ratio predictions should account for systematic uncertainties $\left(\sigma_{\text {sys }} \sim 0.3\right.$ dex; e.g. Barro et al. 2011) related to the assumed stellar population models, IMF, SFHs, extinction law, etc. Finally,

$\sigma_{\Upsilon}^{2}=[B+2 C(g-i)]^{2} \sigma_{(g-i)}^{2}+\sigma_{\text {int }}^{2}+\sigma_{\text {sys }}^{2}$.

Our measurements suggest that the estimated MLCRs are redshift-independent, at least since $z \sim 1.5$. That is, quiescent and star-forming galaxies have evolved along the MLCRs in the last 9 Gyrs of the Universe, preserving the observed relations with time.

We compare our data with other proposed MLCRs in the literature. The observational relation of T11, based on GAMA survey data, reproduces our values with no bias and dispersion $\sigma_{\Delta \Upsilon}=0.1$ dex. Regarding theoretical studies, the MLCR from Roediger \& Courteau (2015) matches our measurements most closely; the bias is below $0.1 \mathrm{dex}$ and the dispersion is $\sigma_{\Delta \Upsilon}=0.17$ dex.

Our results could be expanded in several ways. The analysis could be made by using different stellar population models to test the redshift independence of the relations and the curvature of the star-forming MLCR. The study of the MLCR at higher redshifts will provide extra clues about the absence of redshift evolution, for which a NIR-selected ALHAMBRA sample is needed (Nieves-Seoane et al. 2017). Finally, the study at masses lower than $\log _{10} M_{\star} \sim 8$ will test the robustness of the results at the bluer end of the relation, where intense star-forming episodes could compromise the stellar masses estimated with our current techniques.

\footnotetext{
3 To estimate this offset, we directly compared the stellar masses computed by Barro et al. (2011) with PEGASE and BC03 for a set of galaxies in the Extended Groth Strip (available at rainbowx. fis.ucm.es).
}

The derived relations can be used to estimate stellar masses with photometric redshift codes based on a limited set of empirical templates, such as BPZ2. The intrinsic MLCRs, unaffected by observational errors, are the needed priors to define the probability distribution function (PDF) of the stellar mass. The PDF-based estimator of the luminosity function was presented by López-Sanjuan et al. (2017) as part of the PROFUSE ${ }^{4}$ project, which uses PRObability Functions for Unbiased Statistical Estimations in multi-filter surveys, and has been successfully applied to estimate the $B$-band luminosity function at $z<1$ (López-Sanjuan et al. 2017) and the $U V$ luminosity function at $2.5 \leq z<4.5$ (Viironen et al. 2018) in ALHAMBRA. The present paper is a fundamental step towards a PDF-based estimator of the stellar mass function.

Acknowledgements. We dedicate this paper to the memory of our six IAC colleagues and friends who met with a fatal accident in Piedra de los Cochinos, Tenerife, in February 2007, with a special thanks to Maurizio Panniello, whose teachings of python were so important for this paper. We thank the anonymous referee for the comments and suggestions that improved the quality of the results and the readability of the manuscript. We thank R. Angulo, S. Bonoli, A. Ederoclite, C. Hernández-Monteagudo, A. Marín-Franch, A. Orsi, and all the CEFCA staff, post-docs, and students for the useful and productive discussions. This work has been mainly funded by the FITE (Fondos de Inversiones de Teruel) and the Spanish MINECO/FEDER projects AYA2015-66211-C2-1-P, AYA201230789, AYA2006-14056, and CSD2007-00060. We also acknowledge the financial support from the Aragón Government Research Groups E96, E103, and E16_17R. We acknowledge support from the Spanish Ministry for Economy and Competitiveness and FEDER funds through grants AYA2010-15081, AYA201022111-C03-01, AYA2010-22111-C03-02, AYA2012-39620, AYA2013-40609-P, AYA2013-42227-P, AYA2013-48623-C2-1, AYA2013-48623-C2-2, AYA201676682-C3-1-P, AYA2016-76682-C3-3-P, ESP2013-48274, Generalitat Valenciana project Prometeo PROMETEOII/2014/060, Junta de Andalucía grants TIC114, JA2828, P10-FQM-6444, and Generalitat de Catalunya project SGR1398. K.V. acknowledges the Juan de la Cierva incorporación fellowship, IJCI-2014-21960, of the Spanish government. A.M. acknowledges the financial support of the Brazilian funding agency FAPESP (Post-doc fellowship process number 2014/11806-9). B.A. has received funding from the European Union's Horizon 2020 research and innovation programme under the Marie Sklodowska-Curie grant agreement No. 656354. M.P. acknowledges financial support from the Ethiopian Space Science and Technology Institute (ESSTI) under the Ethiopian Ministry of Science Science and Technology (MoST). This research made use of Astropy, a community-developed core Python package for Astronomy (Astropy Collaboration 2013), and Matplotlib, a 2D graphics package used for Python for publication-quality image generation across user interfaces and operating systems (Hunter 2007).

\section{References}

Abazajian, K. N., Adelman-McCarthy, J. K., Agüeros, M. A., et al. 2009, ApJS, 182,543

Aparicio-Villegas, T., Alfaro, E. J., Cabrera-Caño, J., et al. 2010, AJ, 139, 1242 Arnalte-Mur, P., Martínez, V. J., Norberg, P., et al. 2014, MNRAS, 441, 1783 Astropy Collaboration (Robitaille, T. P., et al.) 2013, A\&A, 558, A33 Azzalini, A. 2005, Scand. J. Stat., 32, 159

Barro, G., Pérez-González, P. G., Gallego, J., et al. 2011, ApJS, 193, 30 Bell, E. F., \& de Jong, R. S. 2001, ApJ, 550, 212

Bell, E. F., McIntosh, D. H., Katz, N., \& Weinberg, M. D. 2003, ApJS, 149, 289 Benítez, N. 2000, ApJ, 536, 571

Bongiorno, A., Schulze, A., Merloni, A., et al. 2016, A\&A, 588, A78

Bruzual, G., \& Charlot, S. 2003, MNRAS, 344, 1000

Calzetti, D., Armus, L., Bohlin, R. C., et al. 2000, ApJ, 533, 682

Chabrier, G. 2003, PASP, 115, 763

Chang, Y.-Y., van der Wel, A., da Cunha, E., \& Rix, H.-W. 2015, ApJS, 219, 8 Conroy, C., \& Gunn, J. E. 2010, ApJ, 712, 833

Conroy, C., Gunn, J. E., \& White, M. 2009, ApJ, 699, 486

Courteau, S., Cappellari, M., de Jong, R. S., et al. 2014, Rev. Mod. Phys., 86, 47 Cristóbal-Hornillos, D., Aguerri, J. A. L., Moles, M., et al. 2009, ApJ, 696, 1554 Díaz-García, L. A., Cenarro, A. J., López-Sanjuan, C., et al. 2015, A\&A, 582, A14

4 profuse.cefca.es 
C. López-Sanjuan et al.: The ALHAMBRA survey. Optical mass-to-light ratio versus colour up to $z=1.5$

Díaz-García, L. A., Cenarro, A. J., López-Sanjuan, C., et al. 2017, A\&A, submitted, [arXiv:1711.10590]

Díaz-García, L. A., Cenarro, A. J., López-Sanjuan, C., et al. 2018, A\&A, submitted, [arXiv:1802.06813]

Driver, S. P., Hill, D. T., Kelvin, L. S., et al. 2011, MNRAS, 413, 971

Fioc, M., \& Rocca-Volmerange, B. 1997, A\&A, 326, 950

Fitzpatrick, E. L. 1999, PASP, 111, 63

Foreman-Mackey, D., Hogg, D. W., Lang, D., \& Goodman, J. 2013, PASP, 125, 306

Gallazzi, A., \& Bell, E. F. 2009, ApJS, 185, 253

Gallazzi, A., Charlot, S., Brinchmann, J., White, S. D. M., \& Tremonti, C. A 2005, MNRAS, 362, 41

Gallazzi, A., Bell, E. F., Zibetti, S., Brinchmann, J., \& Kelson, D. D. 2014, ApJ 788,72

Goodman, J., \& Weare, J. 2010, Comm. App. Math. Comp. Sci., 5, 65

Herrmann, K. A., Hunter, D. A., Zhang, H.-X., \& Elmegreen, B. G. 2016, AJ, 152,177

Huertas-Company, M., Bernardi, M., Pérez-González, P. G., et al. 2016, MNRAS, 462, 4495

Hunter, J. D. 2007, Comput. Sci. Eng., 9, 90

Ilbert, O., Lauger, S., Tresse, L., et al. 2006, A\&A, 453, 809

Into, T., \& Portinari, L. 2013, MNRAS, 430, 2715

Jablonka, J., \& Arimoto, N. 1992, A\&A, 255, 63

Kauffmann, G., Heckman, T. M., White, S. D. M., et al. 2003, MNRAS, 341, 54

Lara-López, M. A., Cepa, J., Bongiovanni, A., et al. 2010, A\&A, 521, L53

López-Sanjuan, C., Tempel, E., Benítez, N., et al. 2017, A\&A, 599, A62

Mannucci, F., Cresci, G., Maiolino, R., et al. 2009, MNRAS, 398, 1915

Maraston, C. 2005, MNRAS, 362, 799

McGaugh, S. S., \& Schombert, J. M. 2014, AJ, 148, 77
Moffett, A. J., Ingarfield, S. A., Driver, S. P., et al. 2016, MNRAS, 457, 1308

Moles, M., Benítez, N., Aguerri, J. A. L., et al. 2008, AJ, 136, 1325

Molino, A., Benítez, N., Moles, M., et al. 2014, MNRAS, 441, 2891

Montero-Dorta, A. D., Bolton, A. S., Brownstein, J. R., et al. 2016, MNRAS, 461, 1131

Nieves-Seoane, L., Fernandez-Soto, A., Arnalte-Mur, P., et al. 2017, MNRAS, 464, 4331

Noeske, K. G., Weiner, B. J., Faber, S. M., et al. 2007, ApJ, 660, L43

Oke, J. B., \& Gunn, J. E. 1983, ApJ, 266, 713

Portinari, L., Sommer-Larsen, J., \& Tantalo, R. 2004, MNRAS, 347, 691

Roediger, J. C., \& Courteau, S. 2015, MNRAS, 452, 3209

Schwarz, G. 1978, Ann. Statist., 6, 461

Shen, S., Mo, H. J., White, S. D. M., et al. 2003, MNRAS, 343, 978

Taylor, E. N., Hopkins, A. M., Baldry, I. K., et al. 2011, MNRAS, 418, 1587

Taylor, E. N., Hopkins, A. M., Baldry, I. K., et al. 2015, MNRAS, 446, 2144

Tinsley, B. M. 1981, MNRAS, 194, 63

Tremonti, C. A., Heckman, T. M., Kauffmann, G., et al. 2004, ApJ, 613, 898

Trujillo, I., Conselice, C. J., Bundy, K., et al. 2007, MNRAS, 382, 109

van de Sande, J., Kriek, M., Franx, M., Bezanson, R., \& van Dokkum, P. G. 2015, ApJ, 799, 125

van der Wel, A., Franx, M., van Dokkum, P. G., et al. 2014, ApJ, 788, 28

Vazdekis, A., Koleva, M., Ricciardelli, E., Röck, B., \& Falcón-Barroso, J. 2016, MNRAS, 463, 3409

Viironen, K., López-Sanjuan, C., Hernández-Monteagudo, C., et al. 2018, A\&A, 614, A129

Williams, R. J., Quadri, R. F., Franx, M., van Dokkum, P., \& Labbé, I. 2009, ApJ, 691, 1879

Zaritsky, D., de Gil Paz, A., \& Bouquin, A.Y.K. 2014, ApJ, 780, L1

Zibetti, S., Charlot, S., \& Rix, H.-W. 2009, MNRAS, 400, 1181 